\title{
FAUT-IL EN FINIR AVEC LA PLURALITÉ DES ORIENTATIONS EN PSYCHOTHÉRAPIE
}

\author{
Nicolas DURUZ ${ }^{1}$
}

\footnotetext{
${ }^{1}$ Psychologue-psychothéraeute FSP, responsable de l'Unité d'Enseignement - Centre d'Etude de la Famille et Codirecteur de l'Institut Universitaire de Psychothérapie au Département de Psychiatrie à Lausanne. Professeur à l'Institut de psychologie de l'Université de Lausanne (BFSH 2, Dorigny, CH - 1015 Lausanne. E-mail: Nicolas.Duruz.@unil.ch).
} 


\section{FAUT-IL EN FINIR AVEC LA PLURALITE DES ORIENTATIONS EN PSYCHOTHERAPIE ?}

La pluralité de méthodes psychothérapeutiques n'est pas sans générer plusieurs problèmes dont, entre autres, des rivalités et des disqualifications mutuelles. Selon l'auteur, divers courants de pensée cherchent à unifier le champ de la psychothérapie, mais sans y parvenir vraiment.

Il émet alors l'hypothèse que ces méthodes sont sur certains points irréductibles les unes aux autres en fonction de leur «philosophie» ou de leurs présupposés. Pour ces méthodes considérées comme autant de «cultures» psychothérapeutiques spécifiques, il propose néanmoins des critères rigoureux en vue de leur reconnaissance scientifique. Il les invite par ailleurs à un dialogue sur la base d'une «charte épistémologique » à rédiger. Dialogue nécessairement tensionnel, à l'abri aussi bien d'un esperanto que d'un despotisme thérapeutiques !

\section{Mots-clés :}

Pluralité des psychothérapies - Anthropologie clinique - Epistémologie - Croyance

\section{SHOULD WE DO AWAY WITH MULTIPLE PSYCHOTHERAPY APPROACHES ?}

Plurality creates rivalry and mutual disqualification. According to the author, several trends try to unify the psychotherapy field without really succeeding.

The author sees these methods as irreconcilable on specific dimensions because of philosophical diversity and multiple assumptions. He proposes rigorous criteria to scientifically validate these methods, considering these as specific psychotherapeutic « cultures ». He finally calls for an epistemological credo of each approach, a way of sustaining openness between them, albeit with friction, but limiting what could be called a therapeutic Esperanto as well as therapeutic despotism !

\section{$\underline{\text { Key-words : }}$}

Psychotherapy plurality - Clinical anthropology - Epistemology - Belief 


\section{FAUT-IL EN FINIR AVEC LA PLURALITÉ DES ORIENTATIONS EN PSYCHOTHÉRAPIE}

Dans le deuxième volume de la revue Psychothérapies, datant de 1982, j'ai rédigé un article intitulé : «Psychothérapies : Une pluralité inquiétante? (Duruz, 1982). Presque 25 ans plus tard, je me retrouve avec la même préoccupation, tout aussi intense, ayant eu toutefois l'occasion entre-temps de mieux comprendre les tenants et aboutissants de cette pluralité et de développer quelques outils de dialogue dans le cadre de mes recherches en psychothérapie comparée. C'est ce que je souhaite développer dans cette conférence.

\section{La pluralité des psychothérapies mise en question}

Plus les années passent et plus je fais le constat, en tant qu'enseignant, psychothérapeute rencontrant des patients, mais aussi comme observateur du mouvement de la psychothérapie, d'une attitude de réticence, d'interrogation, voire d'agacement, envers la pluralité des orientations en psychothérapie. Je le remarque d'abord dans la nouvelle génération des psychiatres assistants. Dans le cadre d'un cours d'introduction à la psychothérapie, dont je suis responsable et qui leur est adressé durant la première et seconde années de leur formation dans le but de les aider à choisir une des orientations prévues dans le règlement pour le titre de psychiatre et psychothérapeute FMH (thérapies psychanalytique, systémique ou cognitivecomportementale), ils sont toujours nombreux à questionner la nécessité de ce choix et l'utilité de plusieurs approches psychothérapeutiques. Certains disent mal percevoir les différences théoriques qui les séparent et doutent qu'elles existent pour le bien des patients.

Sans doute, ces jeunes collègues travaillent dans un cadre où j'ai le sentiment qu'ils côtoient de moins en moins de médecins-chefs au profil psychothérapeutique aiguisé, s'adonnant eux-mêmes à la psychothérapie lege artis. Mais il y a plus, ils travaillent dans des institutions soumises à des pressions folles de la société. Je veux dire que la fonction sociale de la psychiatrie, qui a toujours été de contribuer au contrôle des désordres sociaux, doit s'exercer aujourd'hui dans des conditions particulièrement difficiles : il lui est demandé d'avoir des réponses à des problèmes qui ne sont de loin pas que psychiatriques (chômage, pauvreté, migration, violence, etc), avec des moyens limités, en vue de résultats qui doivent être efficaces à court terme, évaluation à l'appui. Dans ce contexte d'hyperpragmatisme, 
même si elle peut être souhaitée par certains comme un supplément d'âme à la pratique de soins immédiats et de base, la psychothérapie est perçue par d'autres comme un luxe, particulièrement lorsqu'il s'agit de prendre du temps pour faire une bonne indication en fonction des différentes orientations.

Une autre source de questionnement autour de la pluralité des méthodes en psychothérapie remonte à ses usagers, à leurs proches, comme aux instances sociales qui doivent la réguler. Pensons aux patients et aux associations d'usagers de soins, qui en quête d'une aide psychothérapeutique sont souvent perturbés face aux différentes propositions de traitement. Laquelle choisir? A qui se fier dans un tel marché ? Un même questionnement embarrassant habite le tiers public, qu'il s'agisse des autorités gouvernementales ou des responsables de caisses-maladie. Chacun se demande quelles sont les formes de psychothérapie sérieuses, dites scientifiques, qu'il convient de reconnaître et dont les prestations méritent d'être remboursées.

Enfin, je relèverais un certain énervement, voire un ras-le-bol que suscitent chez plusieurs d'entre nous et d'autres psychothérapeutes non présents ici les luttes intestines entre certaines orientations, luttes qui, selon moi, ne peuvent pas être simplement mises au compte d'un débat franco-français. En effet, que d'énergies dépensées, que de blessures personnelles souvent difficilement cicatrisables, que de suspicion ainsi générée à l'égard de la psychothérapie chez les acteurs sociaux extérieurs à ces combats, si prompts parfois à l'assimiler à du charlatanisme. «Abandonnons nos différences, peut-on entendre, ou voyons plutôt ce qui nous réunit : nous serons ainsi plus forts entre nous, pour le patient et face à ceux qui contestent la psychothérapie ». Même si c'est un appel qui fait sens, je n'y souscris pas entièrement. Je questionnerai cette position, après avoir esquissé les tentatives d'unification dans le mouvement de la psychothérapie, qui veulent précisément parer aux effets délétères de trop de diversité.

\section{Des essais d'unification dans le champ de la psychothérapie}

Avec le dessein plus ou moins explicite de faire face à cette fragmentation inquiétante des formes de psychothérapie et aux menaces qu'elle représente pour tous les acteurs concernés, quatre tentatives ou courants de pensée peuvent être relevés dans l'histoire de la psychothérapie. A leur manière, ils ont cherché ou cherchent à limiter cette pluralité 
inquiétante. J'examinerai successivement et brièvement : l'éclectisme pragmatique, le courant intégrationniste, celui des facteurs communs et celui de la pensée d'école.

Avant de le faire, j'aimerais juste resituer l'exercice pluriel de la psychothérapie et les tentatives d'unification auxquelles il donne lieu, dans le contexte global de notre société occidentale. Celle-ci, on le sait trop, est caractérisée par cette même fragmentation de ses savoirs et de ses pratiques, phénomène que certains sociologues du politique attribuent entre autre à l'évolution de notre régime démocratique de nature très individualiste (Gauchet, 1985). Pensons seulement aux diverses possibilités données aujourd'hui de vivre en famille, de pratiquer sa sexualité, de procréer, ou encore à l'émergence de nouveaux champs de savoir ou corps de métier toujours plus hyperspécialisés, pour ne parler que de ces domaines restreints. Face à cette fragmentation galopante qui, par le développement de l'esprit de concurrence et d'individualisme qu'elle génère, porte directement atteinte au lien social, on peut observer au niveau de notre société occidentale plusieurs mouvements de fond qui tentent eux aussi de maîtriser ce processus de morcellement. Mais leur capacité à rassembler les hommes semble limitée, à la source même parfois d'effets perturbateurs non souhaités. La science, formidable entreprise pour fédérer les hommes sous la bannière de la raison, en proposant de nouvelles certitudes en lieu et place de celles offertes par le discours « au nom de Dieu », se trouve contestée par le relativisme de l'idéal post-moderne. L'arsenal du monde technique, capable de réunir anonymement les humains, contribue en même temps à la production d'un homme «unidimensionnel». On pourrait évoquer aussi les grands rassemblements de foule lors de fêtes sportives ou musicales, mais ce ne sont là que des succédanés passagers. Ou encore les nouveaux montages psycho-religieux, suscités par un certain « retour du religieux » dans une société laïque, mais dont les bricolages ésotériques ont de la peine à épouser des formes institutionnelles largement reconnues.

Science d'une action humaine, la psychothérapie en tant que pratique sociale est donc soumise au même morcellement. Des tentatives d'unification sont ainsi également à l'œuvre. Commençons par examiner la première des quatre mentionnées, à savoir l'éclectisme pragmatique. Celui-ci consiste à recourir à un ensemble de techniques provenant d'écoles ou d'orientations différentes, avec une recherche essentiellement centrée sur l'efficacité thérapeutique, sans le souci de les inscrire dans un modèle théorique. D'après de nombreuses enquêtes, un grand nombre de psychothérapeutes y souscrit (entre 40 et $60 \%$ ), en alléguant la diversité des difficultés de leurs patients, lesquelles réclament une diversité de stratégies pour 
être solutionnées. Apportons une première nuance : se déclarer éclectique, ouvert à d'autres techniques que celles de son orientation de base à laquelle on continue de se référer, est une position assez différente de celle qui consiste à accumuler le plus grand nombre de techniques, pour être efficace avec le plus grand nombre de ses patients, indépendamment de ce que ces techniques engagent dans la relation thérapeutique. En outre, l'éclectisme pragmatique est porteur d'un certain discrédit à l'égard du statut scientifique de la psychothérapie (avec le pressentiment qui, selon moi, n'est pas faux, que la science est source de division) et renforce en conséquence un recours à des gestes professionnels très technicisants.

Le courant intégrationniste, dont on trouvera une très bonne présentation dans l'ouvrage de Norcross et Goldfried (1992), ses défenseurs acharnés, se différencie de l'éclectisme pragmatique par un souci d'articuler théoriquement entre elles des stratégies thérapeutiques provenant de différents modèles. Tout en se voulant à l'abri d'une méta-théorie dogmatisante, ce courant cache toutefois mal selon moi le fait que le modèle intégrationniste proposé est souvent construit in fine sous l'influence d'un modèle prévalent, où l' « entrée » cognitiviste, portée par une épistémologie à tendance plus positiviste que critique, est privilégiée.

Le courant des facteurs communs non spécifiques a, quant à lui, une grande tradition. Remontant à Frank (1991), puis défendu par Luborsky (1975), il tend à insister sur les facteurs communs, responsables du processus thérapeutique au détriment de facteurs spécifiques propres à chaque orientation, lesquels seraient moins décisifs dans le processus thérapeutique. Plusieurs études ont pu mettre en évidence que probablement l'efficacité des psychothérapies est due pour une bonne part à des facteurs communs, non spécifiques aux techniques de chaque orientation. Parmi ces facteurs figurent l'alliance thérapeutique, l'attente du patient d'aller mieux, renforcée elle-même par l'expérience qu'il fait en thérapie d'un début de maîtrise de certains de ses comportements problématiques, ou encore, la possibilité d'y vivre une réelle expérience émotionnelle, etc. Des analyses plus fines ont pu montrer toutefois que la spécificité des interventions psychothérapeutiques n'était pas à exclure complètement, car sur la base d'une bonne alliance thérapeutique certaines formes d'intervention ont une efficacité plus affirmée pour certains types de patients avec des troubles psychiques précis. La question des indications revient à la surface, et un nivellement par le bas - toute intervention psychothérapeutique est plus efficace qu'une non intervention est peu stimulante cliniquement et scientifiquement. Par ailleurs, de récentes études 
empiriques à l'Institut Universitaire de Psychothérapie à Lausanne (de Roten, 2005) invitent aussi à ne pas dissocier les facteurs communs des facteurs spécifiques, mais à les penser plutôt en synergie: ces études permettent de faire l'hypothèse que l'établissement de l'alliance est meilleur lorsque le psychothérapeute utilise ses techniques de manière ajustée au fonctionnement psychique de son patient.

Enfin, une dernière manière de maîtriser la pluralité des psychothérapies serait celle développée par la "pensée d'école ». On caractérise par là toute orientation ayant tendance à présenter son modèle, comme le seul et unique qui soit pertinent et efficace, dans la quasiméconnaissance des autres, en s'imposant souvent de manière disqualifiante et arrogante à l'égard des autres orientations. On s'en doute, ce n'est pas là la meilleure manière de régler la question de la pluralité des psychothérapies, et les rivalités ouvertes ainsi engendrées en sont une première raison. Avec l'éclectisme pragmatique, la pensée d'école constitue l'autre pôle extrême de ce continuum réunissant diverses tentatives qui cherchent toutes, à leur manière, à minimiser les différences entre orientations psychothérapeutiques.

\section{La force des présupposés}

Comment expliquer qu'aucun de ces courants de pensée n'arrive finalement à éponger cette diversité des orientations psychothérapeutiques, en s'imposant comme suffisamment fédérateur? Mon hypothèse est la suivante : les différents modèles de psychothérapie sont chacun sous-tendus par des présupposés ou a priori différents, en quelque sorte visions du monde portant sur l'homme, la société, la science, qui influencent la construction de ces modèles et de leurs techniques, au point de les rendre jusqu'à un certain point irréductibles les uns aux autres. Je vais consacrer le reste de mon exposé à préciser ce qu'il faut entendre par présupposé dans le champ de la psychothérapie, ce qui me conduira, engageant mon propre présupposé épistémologique, à soutenir la nécessité d'une pluralité de méthodes psychothérapeutiques. Mais celles-ci, pour être reconnues, doivent toutefois satisfaire à certains critères que je préciserai par ailleurs.

Je tends donc à soutenir la position suivante: les méthodes psychothérapeutiques se différencient entre elles en fonction de différences encore plus fondamentales que celles déjà contenues explicitement dans leur modèle théorique et leurs techniques. Cette pluralité de méthodes serait donc en partie redevable à des présupposés différents qui organisent leur 
modèle et la pratique psychothérapeutique qui en découle. Ces prémisses ou présupposés organisateurs peuvent s'entendre au sens de Bateson (1971, p. 230) comme « un ensemble d'hypothèses ou de prémisses habituelles, implicites dans la relation entre l'homme et son environnement ». Ils fonctionnent comme des a priori épistémologiques implicites, qui interviennent inévitablement dans l'appréhension et la construction de la réalité, et qui ont pour caractéristique de se valider par eux-mêmes en engendrant un effet de croyance, voire d'évidence ${ }^{2}$. Il s'agit donc d'énoncés concernant la psychothérapie qui ne sont pas directement de l'ordre du démontrable, mais de l'ordre de la conviction. Nous avons moins à nous prononcer sur leur vérité ou fausseté qu'à nous déclarer en accord ou en désaccord avec eux. Par exemple, l'énoncé de Carl Rogers : «L'être humain se développe comme la graine du citronnier, grâce aux conditions favorables de son environnement», ou celui de Jay Haley : « Je pense qu'on peut parler à quelqu'un de ses problèmes pendant des années, cela ne changera rien, à moins de faire quelque chose », ou encore celui d'un thérapeute accordant une grande importance à la subjectivité : «La réaction du thérapeute a une valeur diagnostique essentielle: sa subjectivité est un des éléments les plus fiables sur lequel il puisse s'appuyer », de tels énoncés ne relèvent-ils pas davantage d'une adhésion, qu'on peut sans doute plus ou moins raisonner, que d'une démonstration? Dans nos études empiriques auprès de 77 psychothérapeutes suisses de douze orientations différentes (cf. Duruz et Lob, 1997), nous avons pu mettre en évidence trois séries de présupposés de base à la pratique de la psychothérapie, renvoyant à ces trois questions-clés que tout psychothérapeute peut se poser: 1. A quelle image de l'homme se réfère-t-il? (conception optimiste, tragique, adaptative, etc., de l'homme). 2. Dans quel projet de société inscrit-il son activité de psychothérapeute? (une activité qui se veut à l'abri du social, ou qui prend en compte ses dimensions politique, institutionnelle ou économique, etc.). 3. Quelle valeur scientifique accorde-t-il à son activité de psychothérapeute ? (importance donnée à l'expérience singulière et concrète d'un individu, option d'une épistémologie réaliste ou constructioniste ? place accordée aux études empiriques et aux recherches d'efficacité ? par le recours à la seule méthode expérimentale ? etc.).

\section{Science et croyance}

\footnotetext{
${ }^{2}$ Pour un plus large développement de ce terme et de ses conséquences dans la pratique psychothérapeutique et le dialogue entre psychothérapeutes, cf. Duruz, 1994 (particulièrement pp. 190-196).
} 
A ce stade de l'analyse, il faut faire une pause pour clarifier un point extrêmement important: les présupposés du psychothérapeute, dont je parle, ne relèvent pas seulement d'un processus cognitif; ils engagent son affectitivité, la passion de ses préjugés. Nos préjugés ou présupposés ne sont pas assimilables à de simples costumes du soir, qu'on peut porter ou déposer sans autre. Ils ressortissent à des croyances, non entendues ici dans une acception cognitiviste, à savoir des représentations qui n'auraient pas encore fait l'objet d'une preuve scientifique, et que l'arbitrage de la science pourrait distinguer de la vraie connaissance. Une croyance, ça nous engage toujours. Si nous y tenons, c'est parce qu'elle nous tient dans notre identité. Nous y engageons ce qui nous est le plus cher, au plus près de l'étymologie du verbe anglais to believe, qui dans le vieil anglais du XVI ème siècle signifiait aimer, chérir, considérer comme cher, ce même sens que l'on retrouve dans le mot allemand belieben, et selon la même racine dans le mot latin libet (il plaît) ou libido (plaisir). Une croyance qui nous met au service de, et qui ne porte pas d'abord sur un jugement d'existence, comme on l'entend souvent aujourd'hui dans l'expression «croire en Dieu ». C'est dans ce contexte que le philosophe anglais Bacon pouvait évoquer en 1625 la croyance du vrai comme « jouissance », par opposition au questionnement ou à la recherche de la vérité.

Le terme de croyance est donc plus fort que celui de présupposé, dans la mesure où il implique explicitement des valeurs qu'on investit ou que l'on conteste, autour desquelles on se réunit et pour lesquelles on est prêt à se battre. La passion est alors de mise, nourrie par l'idéalisation narcissique de son objet d'amour' ${ }^{3}$. C'est pour cette raison que chaque orientation est guettée par le danger d'ostracisme, mue qu'elle est par la prétention narcissique à être supérieure aux autres et à pouvoir surmonter les troubles psychiques traités sans succès par celles-ci.

\footnotetext{
${ }^{3}$ La référence ici au narcissisme, pour comprendre la dynamique à l'œuvre dans la pluralité des orientations psychothérapeutiques, peut paraître assez surprenante. Elle s'éclairera si je mentionne le passage, dans mes intérêts de recherche, du thème du narcissisme et de ses pathologies à celui de la psychothérapie comparée. En effet, à partir d'une conception du narcissisme tensionnel, pensé comme une structure de désir de l'homme, qui rend possible son individuation au prix de sa division (c'est grâce à des représentations de soi idéalisées contreinvesties, au détriment d'autres désinvesties consciemment, existantes néanmoins parce qu'investies consciemment, qu'il parvient à dire «moi-je »), j'en suis venu à concevoir les différentes orientations de psychothérapie comme autant de méthodes de changement, qui valorisent explicitement, au nom de leur « narcissisme », un ensemble de dimensions de l'existence humaine, au détriment d'autres qu'elles tendent alors à écarter, voire à méconnaître. Par exemple, la thérapie d'orientation cognitive-comportementale est construite à partir d'une représentation de l'homme qui maîtrise et contrôle son existence, alors que la psychanalyse freudienne promeut une image d'un être humain, livré au tragique de son existence, dont les tenants et aboutissants lui échapperont toujours.
} 
Le Livre noir de la psychanalyse (Meyer, 2005), paru l'année dernière, est criblé de jugements passionnels, parfois quasi diffamatoires, à l'égard de la psychanalyse, jugements au nom de présupposés implicites qu'on est en droit de ne pas partager : centrage sur le symptôme, visée adaptative du traitement, insistance à constituer des faits sur la base d'une observation neutre et objective, etc. Par ailleurs, la réponse d'un psychanalyste, très tranchant dans sa manière de trier le bon grain de la psychanalyse de l'ivraie des psychothérapies, conçues ironiquement comme de la direction de conscience, manifeste à l'envi qu'il est tout aussi impensable pour ce dernier qu'on ne partage pas ses valeurs, auquel cas on est alors réduit à être un « vau-rien ». Je lis : «A refuser la vérité de la condition humaine - il n'y a pas pas de savoir propre à assurer ma jouissance - on s'engage dans ... la psychothérapie, soit le bricolage apte à me donner un billet pour participer au concert universel, ce qui peut certes ne pas paraître négligeable. Encore que, lorsqu'on voit le prix d'entrée payé par les concepteurs de ce livre, le niveau d'argumentation auquel les engage une adresse de« bon sens » à un large public - au niveau bac +5 - on se prend à croire qu'il y a parfois à gagner en restant malade $»$.

Ma propre épistémologie - je suis conscient que j’engage ici un présupposé - consiste à distinguer la connaissance obtenue par la science de celle obtenue par la croyance, mais pour mieux les mettre en relation dans leur interinfluence. Dans la croyance, la connaissance provient d'une adhésion à un objet de foi ou de conviction, alors que dans le premier type de connaissance (la science) l'attitude critique et réflexive qui la caractérise exige l'administration d'une preuve. Grâce à une méthode rigoureuse, définissable dans ses principales opérations de pensée, le sujet connaissant cherche à objectiver étymologiquement, à jeter devant lui ; plus rigoureusement, à construire un objet avec - ce qui se manifeste de la réalité étudiée. Même si l'articulation entre ces deux niveaux de connaissance n'est pas simple, une telle distinction mérite d'être faite pour mieux saisir leur influence réciproque. En ce sens, notre épistémologie se différencie du rationalisme critique de Karl Popper qui voudrait que tout scientifique fasse l'effort nécessaire pour se dégager des croyances qui le guettent et dont il parviendrait à se dépouiller en soumettant ses énoncés aux procédures de falsifiabilité. Dans une visée moins rationaliste, nous pensons que ces croyances sont inhérentes au modèle, qu'il ne s'agit pas comme scientifique de s'en défaire, mais de reconnaître, plus positivement, qu'elles rendent possible sa construction, le marquant 
certes d'une certaine partialité, mais fondant par-là même l'efficacité de ses limites ${ }^{4}$. Par ailleurs, ce sont ces croyances plutôt que d'autres qui font que certains psychothérapeutes se retrouvent regroupés dans une orientation et au nom desquelles ils l'ont choisie.

La passion violente du narcissisme est intolérante, ostraciste, sectaire, nourrit le sentiment d'être unique et le meilleur. Quel travail accomplir pour parvenir à ce que Freud appelait le « narcissisme des petites différences », un éthicien américain la convivialité des « désaccords raisonnables », ou dans mes termes, un dialogue nécessairement tensionnel ?

\section{Un travail critique de clarification}

Pour aller dans ce sens, il me semble qu'un double travail critique doit être opéré par toute école psychothérapeutique et par les psychothérapeutes qui s'y réfèrent, en vue d'une reconnaissance de leur méthode par la communauté scientifique et politique : d'une part, rendre compte de leur modèle selon des critères scientifiques de pertinence et de preuve; d'autre part, expliciter l'épistémologie engagée par leur modèle, de manière à mettre en évidence les croyances et les valeurs spécifiques qui les sous-tendent, et qui ne sont pas nécessairement partagées par d'autres modèles.

Pour la première tâche, nous proposons cinq critères en fonction desquels toute méthode devrait pouvoir être évaluée. Chacune d'elles devrait être capable de :

- $\quad$ rendre compte de ses filiations thérapeutiques et de son contexte socio-culturel d'émergence ;

- $\quad$ présenter une théorie cohérente de la personnalité où sont définis des critères de santé et de pathologie psychiques ;

- dégager les facteurs curatifs du processus thérapeutique, qui permettent de faire un lien entre sa théorie étiopathogénique du trouble psychique, les techniques utilisées et le changement qu'elle vise. ;

- $\quad$ mettre en évidence l'efficacité de sa méthode, sur la base de critères qui lui sont propres ;

\footnotetext{
${ }^{4}$ On peut citer ici cette remarque qu'un ingénieur d'Ecole Polytechnique, romancier à ses heures, met dans la bouche d'un de ses personnages en train de mener une enquête policière au Vatican : «Un modèle n'est utilisable que dans la mesure où il néglige une partie de la réalité, celle que vous décrétez inutile et encombrante pour mener à bien votre raisonnement et votre calcul » (Neyrinck, 1999, p. 131) !
} 
- $\quad$ attester de son insertion sociale dans la communauté scientifique de par ses appartenances institutionnelles, ses activités de formation, de recherche, et de publication.

Comme on le remarque, l'évaluation de la psychothérapie du point de vue de son efficacité ne représente qu'un aspect parmi d'autres.

Pour ce qui est du travail d'explicitation épistémologique, on pourrait souhaiter que chaque orientation et ses psychothérapeutes rédigent une sorte de " charte épistémologique », mettant en évidence les présupposés fondamentaux concernant l'homme tel qu'ils le rencontrent dans le traitement psychothérapeutique, la société telle qu'ils la convoquent dans leur cabinet, et enfin la science, au nom de laquelle ils revendiquent ne pas être des charlatans. Ce serait une manière de répondre à l'appel lancé par Bateson $(1979$, p. 31) au terme de sa vie, si conscient que tout modèle véhicule une certaine anthropologie : «Il est évidemment souhaitable (mais non absolument nécessaire), écrivait-il, que l'homme de science soit conscient de ses présuppositions, et qu'il soit capable de les formuler. Pour pouvoir porter un jugement scientifique, de toute façon, il est recommandé, pour ne pas dire indispensable, de connaître les présuppositions des collègues faisant des recherches dans le même domaine ».

Il faudrait sans doute aller plus loin qu'un seul dialogue au niveau d'un échange de chartes, mais porter ce dialogue jusqu'à une confrontation des pratiques elles-mêmes, selon un modèle que j'ai présenté ailleurs (cf. Duruz, 2002, pp. 6-8).

Mais ne nous le cachons pas, ce travail de clarification épistémologique est extrêmement difficile, puisqu'il engage un travail identitaire concernant des groupes et des individus. Il invite à rendre explicite ce qui par définition est implicite dans le monde de la science, pour nous faire éprouver, comme nous le rappelle cette grande psychanalyste qu'est Mc Dougall (1988, p.606), les limites de chacun de nos modèles : « Nous avons besoin de nous assurer, écrit-elle, qu'il existe un ordre dans le chaos du fonctionnement psychique et qu'il y a des théories pour expliquer les changements psychiques. De plus, nos théories nous aident à faire face aux inconnus de la situation clinique ainsi qu'à nous prémunir contre la solitude qui est la nôtre dans cette situation à deux. En nous attachant à une école théorique, nous faisons partie d'une famille, nous sommes moins seuls face aux incertitudes qui nous assaillent tous 
les jours. (...) L'idéal serait de tenir dans le même respect que les nôtres les théories des autres ; cela nous permettrait de mieux percevoir les limites de nos propres modèles et leur prégnance ».

Le réel dialogue entre psychothérapeutes repose selon moi sur le repérage de leurs présupposés compatibles et incompatibles, grâce auquel chacun est censé pouvoir situer sa méthode dans une articulation épistémologique à celles des autres, voire évoluer dans ses propres présupposés.

\section{L'apport de l'anthropologie clinique}

Pour favoriser ce dialogue entre psychothérapeutes, j'essaie depuis quelques années de promouvoir ou plutôt de redonner de l'actualité au mouvement de l'anthropologie clinique. Celui-ci, chevillé dans le courant de la phénoménologie et porté tout particulièrement par quatre grands psychiatres-philosophes du milieu du $20^{\text {ème }}$ siècle (Ludwig Binswanger, Eugen Minkowski, Erwin Straus et Viktor von Gebsattel ${ }^{5}$ ), a cherché à constituer une psychiatrie scientifique qui ne fasse pas pour autant l'économie de l'expérience vécue du patient, telle que l'a thématisée de manière très rigoureuse comme expérience corporelle d'être au monde la phénoménologie. Selon l'anthropologie clinique, le patient révèle à travers ses troubles psychiques une expérience d'habiter le monde, qui peut beaucoup nous apprendre sur les formes principales d'être homme et sur son travail d'humanisation (cf. Schotte, 1990). Je ne peux bien sûr pas m'arrêter longuement sur ces apports. Je voudrais seulement dire deux mots de la réduction phénoménologique, procédure de pensée qui, comme on le verra, favorise l'explicitation des préjugés dans une visée comparative. L'établissement d'un dialogue entre différentes orientations psychothérapeutiques peut s'en trouver amélioré.

Quand l'anthropologie phénoménologique nous invite avec Husserl à retourner aux choses elles-mêmes (zu den Sachen selbst), quand celui-ci en vient à mettre l'accent, à la fin de son œuvre surtout, sur l'expérience (Erfahrung) de l'humain en lien avec son Lebenswelt, le monde de la vie (ce que Merleau-Ponty prolongera par son insistance sur une subjectivité incarnée, qui fait que c'est notre corps vécu tout entier qui est esprit et que c'est à partir de lui que nous sommes au monde), n'est-t-on pas invité à toujours chercher quelque chose

\footnotetext{
${ }^{5}$ Dont la pensée s'est prolongée jusqu'à nos jours par l'œuvre, entre autres, de Roland Kuhn, Arthur Tatossian, Georges Lantéri-Laura, Jacques Schotte, Jean Naudin, etc.
} 
d'antéprédicatif, à l'origine de la construction de nos modèles si spécialisés, et à rencontrer ainsi l'homme dans son expérience originaire plutôt que l'homme d'un modèle ? Certes, les modèles sont indispensables mais ils renvoient toujours à un homme abstrait, que ce soit l'abstraction de l'homme moyen de la psychopathologie différentielle, celle de l'homme ratomorphique, pour reprendre une expression de Bertanlanffy, issue du laboratoire et qui oriente de loin certaines formes de thérapie, celle de l'homme neuronal, comme on tend à le dire aujourd'hui, ou même l'abstraction d'un homo psychoanalyticus, tel qu'il peut émerger d'une métapsychologie un peu trop horlogère où les instances psychiques se figent, se substantifient, considérées qu'elles sont comme des instances agentielles.

Pour nous aider à retourner à la chose elle-même en deçà de nos modèles - en ce qui nous concerne, à l'homme en souffrance d'humanisation, à la recherche d'une « repossibilisation » (Vermöglichung) de son existence, actuellement figée dans une forme caricaturale ou excessive d'être homme -, l'anthropologie clinique, d'orientation phénoménologique, nous propose une méthode nommée réduction phénoménologique. Il s'agit d'une opération de la pensée qui vise à suspendre, à mettre entre parenthèses (époché) l'attitude «naturelle » que nous avons envers la réalité, attitude qui nous fait oublier que c'est à travers un regard spécifique sur elle qu'elle nous apparaît telle. Collés à nos modèles, sans les mettre en doute, nous avons tendance à «naturaliser» nos connaissances, alors qu'elles sont toujours une thématisation, bien qu'indispensable, j'insiste à nouveau, d'une réalité antéprédicative ${ }^{6}$. En parlant par exemple de la suspension des thèses métaphysiques sur le monde, qui habitent l'empirisme ou le rationalisme, les approches subjectiviste ou objectiviste, naturaliste ou historiciste, causaliste ou téléologique du monde, Husserl (1927, p. 243) écrit : «On trouve partout des motifs légitimes, mais partout des demi-mesures ou des façons irrecevables de rendre absolues des vues unilatérales qui ne sont légitimes que d'un point de vue relatif et abstrait ». L'époché consiste donc à mettre entre parenthèses ses préjugés d'analyse, à revenir

\footnotetext{
${ }^{6}$ Husserl essaie de montrer que toute connaissance du monde s'acquiert dans l'acte transcendental d'une constitution de sens, où la conscience incarnée, à partir des synthèses passives qu'elle opère et qui l'ouvrent à l'horizon de ce qu'elle appréhende, produit un acte originaire où le monde est « thématisé ». Comme l'écrit S. Giudicelli (2004, p. 103), de manière un peu savante pour les non philosophes mais combien rigoureuse : « Le monde de la vie dont nous partons toujours, et où nous revenons sans cesse, est antéprédicatif. C'est dans cet entrelacement que se structure, dans l'intentionnalité, l'intelligible le plus comlexifié, par esquisses constantes et enrichisement de sens. La recherche de l'explication est cette tension qui prend appui sur le renvoi constant des synthèses passives à l'acte originaire où la subjectivité dans son choix primordial avec la totalité du monde comme donné, s'ébranle dans la genèse active. La recherche de l'explication est ce dépassement de la synthèse passive en synthèse active, qui devient synthèse prédicative. (...) Dans l'activité antéprédicative gîte une préconstitution passive de signification dont la subjectivité constituante incarnée dans le monde de la vie est imprégnée ».
} 
à sa propre subjectivité constituante, dans ce moment originaire de la constitution de sens. Selon la belle expression d'Husserl encore, il s'agit « de faire vœu de pauvreté en matière de connaissance » pour ne pas s'aliéner dans des modèles trop absolus.

Si nous sommes chacun pris par notre méthode psychothérapeutique, en fonction de ses présupposés, qui conceptualisent et actualisent pour nous, et notre client aussi, une manière spécifique d'expérimenter le dysfonctionnement psychique et sa transformation, une manière d'être en relation thérapeutique, une manière de "faire partie" de la société, etc., on comprend aisément les difficultés qui peuvent exister à nous entendre. Incompréhension non seulement entre psychothérapeutes, mais également entre le psychothérapeute et le patient qui ne partage peut-être pas du tout la même vision du monde que son thérapeute, dans sa manière de vivre et de comprendre son trouble. Il est indispensable que le psychothérapeute vérifie toujours ce minimum d'affinités électives existant entre lui et son patient quant à cet implicite de la relation. Cela mérite d'être souligné dans cet exposé qui, il est vrai, est consacré surtout aux effets de la pluralité des méthodes psychothérapeutiques sur les relations entre psychothérapeutes. Nos présupposés, nos préjugés, non explicités, nous font donc voir certaines choses plutôt que d'autres, et qui plus est, génèrent certaines évidences sur notre manière de saisir les troubles psychiques, comme si les limites du modèle nous échappaient, parce que nous échappent les présupposés qui l'animent. Montesquieu avait une jolie définition en parlant du préjugé : j'appelle préjugé non pas ce qui fait que j'ignore des choses, mais ce qui fait que je m'ignore moi-même.

Si l'on réussit à mettre un peu en évidence la relation que nous avons à notre modèle, c'est donc du même coup devenir critique à son égard, découvrir ses limites, devenir capable de référer certains de nos patients à des collègues qui travaillent différemment de nous, mettre un frein à la passion de l'intolérance qui peut surgir quand on se croit être le seul et le meilleur. Et c'est en conséquence, oser aller voir dans le «pré » de l'autre, sachant que ce sera certes différent, mais qu'on pourra peut-être même y voir du neuf. C'est là un autre apport de l'anthropologie clinique, mais sur lequel je ne m'arrêterai pas, et qui relève de sa dimension herméneutique, à savoir qu'on ne peut vraiment se connaître qu'à partir de l'autre et que s'intéresser à l'autre différent de soi contribue à la compréhension de soi-même. En d'autres termes, la connaissance comparée des autres, c'est simultanément la mise en perspective de soi-même. 


\section{Parler plusieurs langues?}

Je vais terminer mon exposé par le recours à la métaphore de la langue et de sa pratique, une manière d'exprimer de manière un peu analogique et légère ce que n'a pas toujours réussi à faire, j'en suis conscient, le phrasé de mes propos parfois un peu arides. La plupart d'entre nous, en tout cas parmi les aînés ici présents, avons été formés selon une orientation psychothérapeutique spécifique, dans laquelle nous avons baigné et qui, dès la mamelle de notre enfance, a imbibé de son idiome maternel nos pensées et nos gestes professionnels. Mais cela n'a pas empêché certains, en fonction de parcours de vie singuliers, de faire l'expérience d'autres cultures et d'autres langues psychothérapeutiques. Cela, de manière prolongée ou brève, imposée ou choisie, prématurée ou tardive. Comment s'en sont-ils trouvés transformés? Il serait intéressant de décrire minutieusement, à la lumière des méthodes de l'anthropologie culturelle et sociale, l'itinéraire de ces psychothérapeutes qui se sont aventurés dans des mondes différents. Quelle est leur identité actuellement? Qu'en est-il de ceux qui se déclarent parfaitement bilingues? de ceux qui ont quasi abandonné une langue pour une autre? de ceux qui, fidèles à leur langue maternelle, se disent enrichis par la pratique d'une ou deux autres langues? ou encore, de ceux qui s'en tiennent à leur première langue, sans en utiliser d'autres tout en s'y montrant curieux et intéressés, voire même de ceux qui privilégient certains dialectes ... ? Souscrivant à une conception ni instrumentale, ni utilitariste du langage, mais davantage ontologique - à savoir, nous sommes toujours immergés en lui et c'est lui qui va façonner cette forme concrète et spécifique que nous aurons d'habiter le monde -, je pense qu'il est dommageable de renoncer à sa langue maternelle, racine de son identité, comme il l'est tout autant de ne pas reconnaître l'existence d'autres langues, ouvrant sur d'autres manières d'habiter le monde. En ce qui concerne la question de la pluralité des psychothérapies, pour le dire brièvement et crûment, deux extrêmes doivent être absolument évités selon moi, qui abolissent l'un et l'autre les différences : l'esperanto psychothérapeutique et les mouvements de purification ethnique-thérapeutique! En fréquentant d'autres langues, on peut découvrir la complexité de la sienne, ses finesses comme ses limites, et se risquer alors à des traductions sans trop de trahison ... Goethe, dans son esprit universel, nous invite à cette ouverture sans renier l'ancrage identitaire de la langue-mère, lorsqu'il écrivait : "Wer nur eine Sprache kennt, kennt keine », ce que je me permets de traduire en ces termes : «Celui qui ne sait qu'une langue, n'en connaît aucune »! 


\section{$\underline{\text { Bibliographie }}$}

BATESON, G. (1971). La cybernétique du « soi » : une théorie de l'alcoolisme. In G. Bateson, Vers une Ecologie de l'Esprit. Tome I, pp. 225-252. Paris : Seuil. Trad. fr. (1977).

BATESON, G. (1979). La nature et la pensée. Paris : Seuil. Trad. fr. (1984).

DE ROTEN, Y., MICHEL, L., DESPLAND, J.-N. (2005). La recherche sur le processus psychothérapeutique : l'exemple du modèle de l'adéquation. Pour la Recherche, 44, 11-13.

DURUZ, N. (1982). Psychothérapies : une pluralité inquiétante ? Psychothérapies, 2/3, $67-74$.

DURUZ, N. (1994). Psychothérapie ou psychothérapies? Prolégomènes à une analyse comparative. Neuchâtel et Paris : Delachaux et Niestlé.

DURUZ, N., GENNART, M. (2002). Traité de psychothérapie comparée. Genève : Médecine et Hygiène.

DURUZ, N., LOB, R. (1997). Psychothérapeutes: analyse de trois présupposés. Psychothérapies, 17, 67-77.

FRANK, J.D., FRANK, J.B. (1991). Persuasion and healing : A comparative study of psychotherapy $\left(3^{\text {nd }}\right.$ ed.). Baltimore : Johns Hopkins University Press.

GAUCHET, M. (1985). Le désenchantement du monde. Une histoire politique de la religion. Paris : Gallimard.

GIUDICELLI, S. (2004). L’implication phénoménologique comme fondement scientifique à la psychothérapie des psychoses. Perspectives Psy, 43, 101-106.

HUSSERL, E. (1927). Psychologie phénoménologique. Paris : J. Vrin. Trad. fr. (2001).

LUBORSKY, L., SINGER, B., LUBORSKY, L. (1975). Comparative studies of psychothérapies : Is it true that "Everyone has won and all must have prizes" ? Archives of General Psychiatry, 32, pp. 995-1008.

MCDOUGALL, J. (1988). Quelles valeurs pour la psychanalyse ? Revue Française de Psychanalyse, 52, pp. 585-612.

MEYER, de C. (sous la dir.) (2005). Le livre noir de la psychanalyse. Paris : Ed. des Arènes.

NEYRINCK, J. (1999). L'ange dans le placard. Paris : Desclée de Brouwer.

NORCROSS, J.T., GOLDFRIED, M.R. (Eds) (1992). Psychothérapie intégrative. Paris : Desclée de Brouwer, 1998. 
SCHOTTE, J. (1990). Comme dans la vie, en psychiatrie... Les perturbations de l'humeur comme troubles de base de l'existence. In J. Schotte, Szondi avec Freud. pp. 173-213. Bruxelles : De Boeck-Wesmael.

\section{$\underline{\text { Adresse de l'auteur : }}$}

Prof. Nicolas Duruz

Institut Universitaire de Psychothérapie

Site de Cery

$\mathrm{CH}-1008$ Prilly / Lausanne

email : Nicolas.Duruz@unil.ch 